\title{
Gordian adjacency for torus knots
}

\author{
PETER FELLER
}

\begin{abstract}
A knot $K_{1}$ is called Gordian adjacent to a knot $K_{2}$ if there exists an unknotting sequence for $K_{2}$ containing $K_{1}$. We provide a sufficient condition for Gordian adjacency of torus knots via the study of knots in the thickened torus $S^{1} \times S^{1} \times \mathbb{R}$. We also completely describe Gordian adjacency for torus knots of index 2 and 3 using Levine-Tristram signatures as obstructions to Gordian adjacency. Our study of Gordian adjacency is motivated by the concept of adjacency for plane curve singularities. In the last section we compare these two notions of adjacency.
\end{abstract}

57M27; 14B07

\section{Introduction}

Let $K_{1}$ and $K_{2}$ be smooth knots in $\mathbb{R}^{3}$ or $S^{3}$. Their Gordian distance $d_{g}\left(K_{1}, K_{2}\right)$ is the minimal number of crossing changes needed to get from $K_{1}$ to $K_{2}$; see eg Murakami [17]. The unknotting number $u(K)$ of a knot $K$, which was already studied by Wendt [25], is the distance $d_{g}(K, O)$, where $O$ denotes the unknot. The Gordian distance induces a metric on the set of (isotopy classes of) all smooth knots. This discrete metric space is huge. For example, every $\mathbb{Z}^{n}$ can be quasi-isometrically embedded into the subspace consisting of all torus knots by a result of Gambaudo and Ghys [8]. In this paper we study the subspace of torus knots and the simple question, 'When is the triangle inequality $d_{g}\left(K_{1}, K_{2}\right) \geq d_{g}\left(K_{2}, O\right)-d_{g}\left(K_{1}, O\right)$ an equality?'

Definition 1 Let $K_{1}$ and $K_{2}$ be knots. We say $K_{1}$ is Gordian adjacent to $K_{2}$, denoted by $K_{1} \leq_{g} K_{2}$, if $d_{g}\left(K_{1}, K_{2}\right)=u\left(K_{2}\right)-u\left(K_{1}\right)$.

Equivalently, a knot $K_{1}$ is Gordian adjacent to $K_{2}$ if $K_{2}$ can be unknotted via $K_{1}$, that is, if there exists a unknotting sequence for $K_{2}$ that contains $K_{1}$. An unknotting sequence for a knot $K$ is a sequence of $u(K)+1$ knots starting with $K$ and ending with the unknot $O$ such that any two consecutive knots are related by a crossing change; see Baader [2]. The name 'Gordian adjacency' is motivated by the connection to algebraic adjacency; see below. Gordian adjacency is a partial order. 
For two coprime natural numbers $n \geq 2$ and $m \geq 2$ we denote by $T(n, m)=T(m, n)$ the (positive) torus knot obtained as the standard closure of the $n$-strand positive braid $\left(\sigma_{1} \cdots \sigma_{n-1}\right)^{m}$ or alternatively as the knot of the singularity $x^{n}-y^{m}$; see Section 6 . The index of a torus knot $T(n, m)$ is the minimum of $n$ and $m$.

Our main results on Gordian adjacency for torus knots are the following.

Theorem 2 Let $(n, m)$ and $(a, b)$ be pairs of coprime natural numbers with $n \leq a$ and $m \leq b$. Then the torus knot $T(n, m)$ is Gordian adjacent to the torus knot $T(a, b)$.

Theorem 3 Let $n$ and $m$ be natural numbers with $n$ odd and $m$ not a multiple of 3 . Then the torus knot $T(2, n)$ is Gordian adjacent to $T(3, m)$ if and only if $n \leq \frac{4}{3} m+\frac{1}{3}$.

The core of the proof of Theorem 2 is a generalization to knots in $S^{1} \times S^{1} \times \mathbb{R}$ of the following elementary fact. If a knot $K$ in $\mathbb{R}^{3}$ has a knot diagram with $n$ crossings, then $u(K) \leq \frac{n-1}{2}$. The proof of Theorem 3 relies on explicit constructions of the required adjacencies and on Levine-Tristram signatures as obstructions to Gordian adjacency.

As a consequence of Theorem 2 Gordian adjacency and Gordian distance for torus knots of a fixed index are completely described, ie if a natural number $a$ is fixed, then

$$
T(a, b) \leq_{g} T(a, c) \text { if and only if } b \leq c
$$

for all $b, c$ coprime to $a$. Hence,

$$
d_{g}(T(a, b), T(a, c))=|u(T(a, b))-u(T(a, c))|=\frac{(a-1)|b-c|}{2},
$$

where the second equation follows from the Milnor conjecture, which determines the unknotting number of torus knots; see (1). For torus knots $T(a, b)$ and $T(c, d)$ of different indices it is in general not clear how Gordian adjacency is characterized in terms of $a, b, c$ and $d$. Theorem 3 provides such a characterization for the case of index 2 and 3 .

Remark 4 To completely determine Gordian adjacency for torus knots of index 2 and 3, additionally to Theorem 3, one has to show that no torus knot of index 3 is adjacent to a torus knot of index 2. More generally, Borodzik and Livingston show that a torus knot cannot be Gordian adjacent to a torus knot of strictly smaller index [4, Lemma 3.1]. Using signature obstructions we can only partially prove this result; see Section 4.

An obvious motivation for finding Gordian adjacencies is that, by definition, every Gordian adjacency determines the Gordian distance of the involved knots. But Gordian 
adjacencies can also lead to good estimates of Gordian distances between nonadjacent torus knots. For example, the adjacencies $T(2,7) \leq_{g} T(2,9)$ and $T(2,7) \leq_{g} T(3,5)$ yield

$$
\begin{aligned}
d_{g}(T(2,9), T(3,5)) & \leq u(T(2,9))-u(T(2,7))+u(T(3,5))-u(T(2,7)) \\
& =4-3+3-2=2 .
\end{aligned}
$$

The converse inequality can be proven using signatures; thus, $d_{g}(T(2,9), T(3,5))=2$. Trying to generalize this example for any two torus knots $T_{1}$ and $T_{2}$ we look for the highest unknotting number $u(K)$ realized by a knot $K$, adjacent to both $T_{1}$ and $T_{2}$, and ask if $u\left(T_{1}\right)-u(K)+u\left(T_{2}\right)-u(K)$ is close to the Gordian distance $d_{g}\left(T_{1}, T_{2}\right)$. An ambitious future goal is to use such Gordian adjacencies to determine Gordian distances between all torus knots up to a constant factor, similarly to what was done for cobordism distance by Baader [3].

The cobordism distance between two knots $K_{1}$ and $K_{2}$ is defined to be the minimal genus of a connected, oriented, and smoothly embedded surface $F$ in $S^{3} \times[0,1]$ with $\partial F=K_{1} \times\{0\} \cup K_{2} \times\{1\}$. Similar to the unknotting number for the Gordian distance, the slice genus or 4-ball genus of a knot, denoted by $g_{s}$, is the cobordism distance to the unknot $O$. As a crossing change can be realized by a cobordism of genus 1 , the Gordian distance is larger than the cobordism distance and a Gordian adjacency between knots $K_{1}$ and $K_{2}$ yields a cobordism of genus $u\left(K_{2}\right)-u\left(K_{1}\right)$.

Another motivation for the study of Gordian adjacency comes from the notion of adjacency for singularities of algebraic curves in $\mathbb{C}^{2}$ studied by Arnold [1], which yields a notion of adjacency for algebraic knots; see Section 6. Such an adjacency of algebraic knots $K_{1}$ and $K_{2}$ yields a smooth algebraic curve $F$ in $\mathbb{C}^{2}$ such that $K_{1}$ and $K_{2}$ are realized as transversal intersection of $F$ with two spheres around the origin of different radii $r_{1}<r_{2}$, ie

$K_{i}=F \cap\left\{(x, y) \in \mathbb{C}^{2} \mid\|x\|^{2}+\|y\|^{2}=r_{i}^{2}\right\} \subset\left\{(x, y) \in \mathbb{C}^{2} \mid\|x\|^{2}+\|y\|^{2}=r_{i}^{2}\right\} \cong S^{3}$.

By a theorem of Kronheimer and Mrowka [13, Corollary 1.3], known as the Thom conjecture, the slice genus $g_{s}\left(K_{i}\right)$ of $K_{i}$ equals the genus of the intersection of $F$ with the ball centered at the origin of $\mathbb{C}^{i}$ of radius $r_{i}$; thus, the cobordism

$$
F \cap\left\{(x, y) \in \mathbb{C}^{2} \mid r_{1}^{2} \leq\|x\|^{2}+\|y\|^{2} \leq r_{2}^{2}\right\}
$$

in

$$
\left\{(x, y) \in \mathbb{C}^{2} \mid r_{1}^{2} \leq\|x\|^{2}+\|y\|^{2} \leq r_{2}^{2}\right\} \cong S^{3} \times[0,1]
$$

has minimal genus $g_{s}\left(K_{2}\right)-g_{s}\left(K_{1}\right)$. By the Milnor conjecture, a consequence of the Thom conjecture, the slice genus and the unknotting number of algebraic knots are 
equal, eg for torus knots one has

$$
u(T(n, m))=g_{s}(T(n, m))=\frac{(n-1)(m-1)}{2}
$$

for all coprime natural numbers $n, m$.

In summary, we know that $u$ and $g_{s}$ coincide on algebraic knots, and both adjacency notions, which could be thought of as relative versions of $u$ and $g_{s}$, respectively, have similar properties. Namely, for both notions it holds that if $K_{1}$ is adjacent to $K_{2}$, then $u\left(K_{1}\right)=g_{s}\left(K_{1}\right) \leq u\left(K_{2}\right)=g_{s}\left(K_{2}\right)$ and the cobordism distance equals $u\left(K_{2}\right)-u\left(K_{1}\right)=g_{s}\left(K_{2}\right)-g_{s}\left(K_{1}\right)$. Furthermore, for both notions $T(n, m)$ is adjacent to $T(a, b)$ if $n \leq a$ and $m \leq b$; see Theorem 2 and Proposition 22. It is then natural to wonder whether the two concepts of adjacency coincide, for example, on torus knots. We answer in the negative in Section 6, but we give a heuristic argument supporting the conjecture that if two torus knots are Gordian adjacent, then they are algebraically adjacent.

To decide whether a knot is Gordian adjacent to another knot, the unknotting numbers of the involved knots should certainly be known; thus, even ignoring the connection to algebraic adjacency, equality (1) is relevant to the study of Gordian adjacency for torus knots. It is used throughout the text.

Section 2 discusses examples of Gordian adjacent torus knots of index 2 and 3. In Section 3 we study unknotting of knots in $S^{1} \times S^{1} \times \mathbb{R}$ and use it to prove Theorem 2 . Section 4 introduces Levine-Tristram signatures as obstructions to Gordian adjacencies and uses them to prove Theorem 3. In Section 5 we study Gordian adjacencies between torus knots of higher indices. The relation between algebraic and Gordian adjacency is discussed in Section 6. In particular, Proposition 23 provides an infinite family of examples of algebraic adjacent torus knots that are not Gordian adjacent.

Acknowledgements I thank Sebastian Baader for introducing me to unknotting and for his ongoing support. Thanks also to Masaharu Ishikawa for enlightening comments and technical references that led to Proposition 23. Finally, I wish to thank the referee for helpful suggestions and corrections. I gladly acknowledge support from the Swiss National Science Foundation (project number 137548).

\section{Examples of Gordian adjacencies}

By definition, the unknot $O$ is adjacent to every knot $K$. Let $k$ be a natural number. The unknotting number of the torus knot $T(2,2 k+1)$ is $k$. An unknotting sequence 
of $T(2,2 k+1)$ is provided by

$$
T(2,2 k+1) \rightarrow T(2,2 k-1) \rightarrow \cdots \rightarrow T(2,5) \rightarrow T(2,3) \rightarrow O .
$$

Consequently, $T(2,2 l+1) \leq_{g} T(2,2 k+1)$ for all $l \leq k$, a simple instance of Theorem 2. We now construct explicit examples of Gordian adjacencies that are not provided by Theorem 2. Let $\lfloor\cdot\rfloor$ denote the integer part of a real number.

Proposition 5 For every natural number $k$ we have

$$
T(2,2 k+1) \leq_{g} T\left(3,\left\lfloor\frac{3}{2} k+1\right\rfloor\right) .
$$

Proof The knot $T(2,2 k+1)$ is the standard closure of the braid

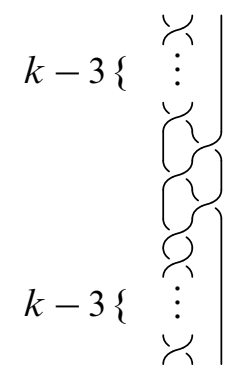

where $k-3$ denotes the number of the crossings not drawn. We introduce a crossing change for knots containing a part that looks (in an appropriate diagram) like the above $T(2,2 k+1)$.

(2)
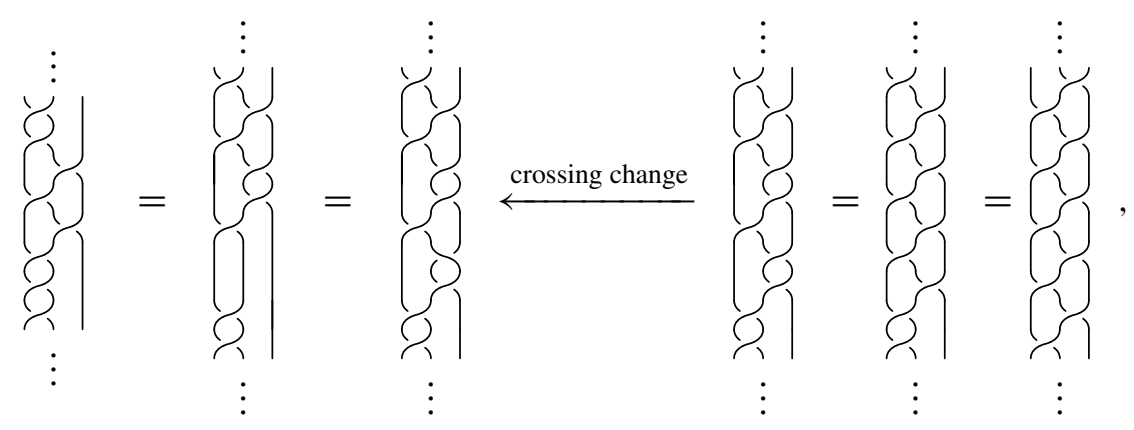

where the first and the two last equalities are obtained by applying the braid relation

$$
\sigma_{2} \sigma_{1} \sigma_{2}=\gamma_{1}^{Y}=\gamma_{1}^{Y}=\sigma_{1} \sigma_{2} \sigma_{1}
$$


First consider the case when $k$ is odd. We use (2) inductively.

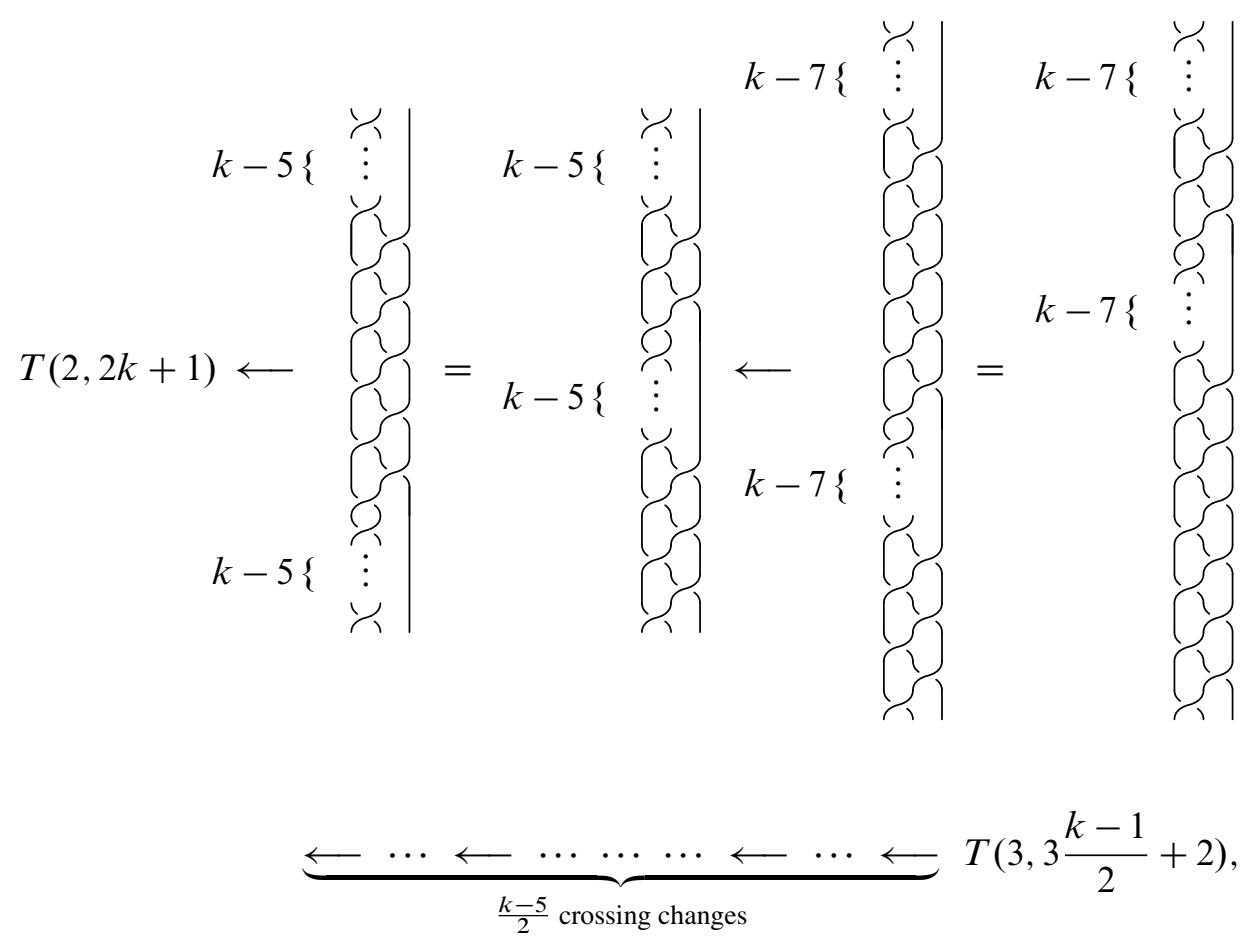

where every arrow indicates a crossing change as in (2). Thus

$$
\begin{aligned}
d_{g}\left(T(2,2 k+1), T\left(3,3 \frac{k-1}{2}+2\right)\right) \leq \frac{k-1}{2} & =\left(3 \frac{k-1}{2}+1\right)-k \\
& \stackrel{(1)}{=} u\left(T\left(3,3 \frac{k-1}{2}+2\right)\right)-u(T(2,2 k+1)) .
\end{aligned}
$$

The case when $k$ is even has essentially the same proof except that the last crossing change does not use (2) but a slight variation of it.

\section{Unknotting on the torus and proof of Theorem 2}

Knots in $\mathbb{R}^{3}$ can be studied via knot diagrams on $\mathbb{R}^{2}$ up to Reidemeister equivalence. Similarly, for a surface $F$ knots in $F \times \mathbb{R}$ can be studied via knot diagrams on $F$.

In a knot diagram on $\mathbb{R}^{2}$ with $n$ crossings one needs to change at most $\left\lfloor\frac{n-1}{2}\right\rfloor$ of the crossings to get the unknot. This is easily proved geometrically by drawing a knot in $\mathbb{R}^{3}$ that projects to the curve on $\mathbb{R}^{2}$ given by the diagram and that descends (or ascends) monotonically except over one point in the diagram (see Figure 1) 

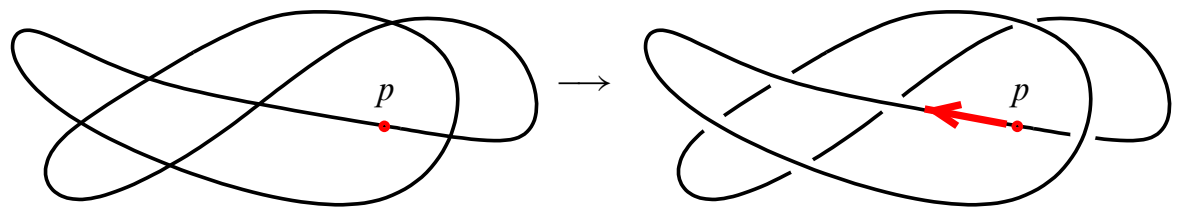

Figure 1: Any curve $c$ in $\mathbb{R}^{2}$ is the projection of the unknot in $\mathbb{R}^{3}$ given by starting at any point $p$ in $\mathbb{R}^{3}$ that projects to $c$ and then descending while following $c$.

and remarking that such a knot is the unknot. To prove Theorem 2, which is a statement entirely about knots in $\mathbb{R}^{3}$, one is surprisingly led to ask whether a similar fact holds for knots in $S^{1} \times S^{1} \times \mathbb{R}$. We provide such a result, which we then use to prove Theorem 2.

Let $F$ be a surface. In what follows a closed smooth curve $c:[0,1] \rightarrow F$ is called presimple if its lift $\widetilde{c}: \mathbb{R} \rightarrow \widetilde{F}$ to the universal cover $\widetilde{F}$ of $F$ is injective and if $c$ is homotopic to a simple closed curve. A knot in $F \times \mathbb{R}$ that is isotopic to a knot that projects to a simple closed curve on $F$ is called unknotted.

Remark 6 There is at most one unknot (up to isotopy) in every homotopy class of closed curves in $F \times \mathbb{R}$. This follows from the fact that homotopic simple closed curves in surfaces are isotopic; see Epstein [7].

In the case of the torus we can be more precise. A homotopy class of closed curves in $S^{1} \times S^{1} \times \mathbb{R}$ contains an unknot, which is unique up to isotopy, if and only if (via the usual identification of $\pi_{1}\left(S^{1} \times S^{1}\right) \cong \pi_{1}\left(S^{1} \times S^{1} \times \mathbb{R}\right)$ with $\left.\mathbb{Z}^{2}\right)$ the corresponding element in $\mathbb{Z}^{2}$ has coprime entries or is $(0,0)$. This is a reformulation of the classification of simple closed curves in $S^{1} \times S^{1}$, written, for example, in Rolfsen's textbook [20].

Lemma 7 For every presimple curve $c$ in $S^{1} \times S^{1}$ there is a knot $O$ in $S^{1} \times S^{1} \times \mathbb{R}$ that projects to $c$ on $S^{1} \times S^{1}$ and that is unknotted.

Remark 8 In terms of knot diagrams Lemma 7 means that if a knot $K$ in $S^{1} \times S^{1} \times \mathbb{R}$ projects to a presimple diagram with $n$ crossings on $S^{1} \times S^{1}$, then one can get the diagram of the unknot by changing at most $\left\lfloor\frac{n}{2}\right\rfloor$ of the $n$ crossings.

To prove this, we use Lemma 7 to get the unknot $O$ with the same diagram as $K$, except it differs in the choice of crossings. If this new diagram differs from the original one in less than half of the crossings, we are done. Otherwise we switch all crossings in the diagram of $O$ yielding a knot diagram of a knot $\bar{O}$. The knot $\bar{O}$ is also unknotted, 
as the following shows. Let $H_{t}$ be an isotopy that changes $O$ to a knot that projects to a simple closed curve on $S^{1} \times S^{1}$. Then parametrize $\bar{O}$ in $S^{1} \times S^{1} \times \mathbb{R}$ exactly the same way as $O$, except changing the sign in the $\mathbb{R}$ coordinate. The same isotopy $H_{t}$ as for $O$ (with a change of sign in the last coordinate) shows that $\bar{O}$ is unknotted.

Clearly the assumption that $c$ is homotopic to a simple closed curve is necessary in Lemma 7. We conjecture that Lemma 7 holds for all curves $c$ that are homotopic to a simple closed curve and, furthermore, that Lemma 7 generalizes to all surfaces.

Proof of Lemma 7 Denote $S^{1} \times S^{1}$ by $F$. Our strategy is to construct a presimple homotopy $h_{t}$ of $c$ (meaning $h_{t}$ is presimple for every $t \in[0,1]$ ) to a simple closed curve and then to find an isotopy $H_{t}$ of knots in $F \times \mathbb{R}$ that has $h_{t}$ as projection.

We first lift the curve $c$ to a mapping $\widetilde{c}: \mathbb{R} \rightarrow \widetilde{F}$, where $\varphi: \widetilde{F} \rightarrow F$ denotes the universal covering map. Since $c$ is presimple, $\widetilde{c}: \mathbb{R} \rightarrow \widetilde{F}$ is injective and there exists a simple closed curve $g:[0,1] \rightarrow F$ that is homotopic to $c$. We take $g$ such that $g(0)=g(1)=c(0)=c(1)$ and denote by $\tilde{g}: \mathbb{R} \rightarrow \widetilde{F}$ its lift to $\widetilde{F}$ with $\tilde{g}(k)=\tilde{c}(k)$ for all $k \in \mathbb{Z}$. Let $\tilde{h}_{t}: \mathbb{R} \rightarrow \widetilde{F}$ be an equivariant ${ }^{1}$ isotopy between $\tilde{c}$ and $\tilde{g}$ that is constant on $\mathbb{Z}$; see Figure 2 .

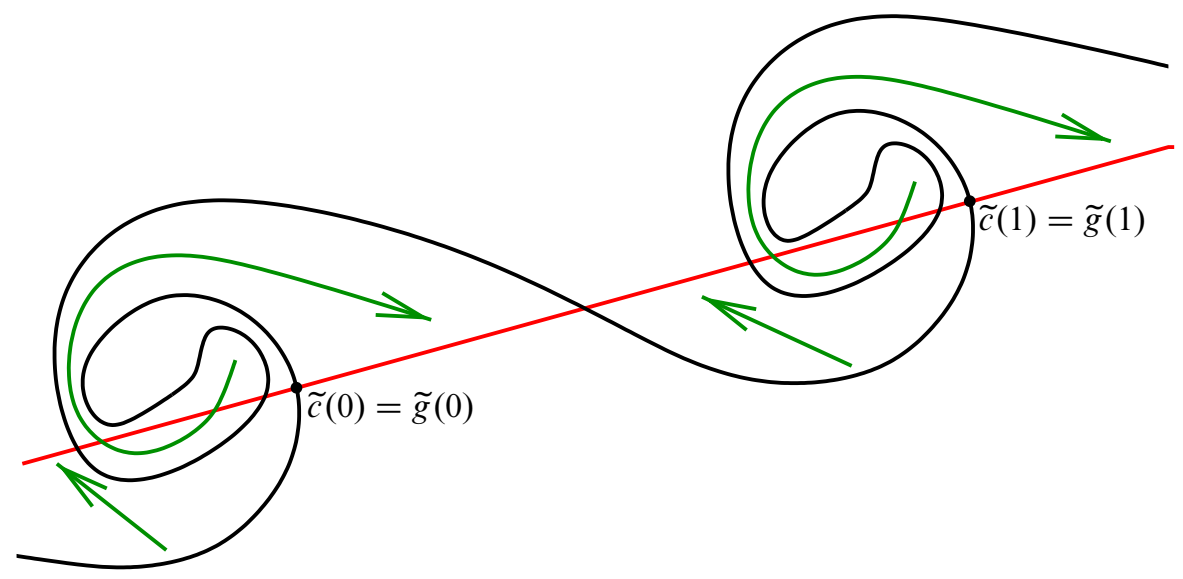

Figure 2: An equivariant isotopy (green) of $\tilde{c}$ (black) to $\widetilde{g}$ (red) is indicated.

Of course $h_{t}=\varphi \circ \tilde{h}_{t}:[0,1] \rightarrow F$ is a presimple homotopy.

The idea for building $H_{t}$ is to measure how far away from $g$ points $p=h_{t}(s)$ are and then to put this distance $d(p)$ in the second coordinate of $H_{t}$. We need a metric to

\footnotetext{
${ }^{1}$ That is $\tilde{h}_{t}(s+1)=D\left(\tilde{h}_{t}(s)\right)$ for all $s$ in $\mathbb{R}$, where $D$ denotes the unique deck transformation sending $\tilde{c}(0)$ to $\tilde{c}(1)$.
} 
make this precise and the distance will actually be measured in the universal cover. Put a Riemannian metric on $F$ with constant curvature 0 such that $g$ is a simple closed geodesic of length 1 . The universal cover $\widetilde{F}$ is identified with the Euclidean plane $\mathbb{R}^{2}$ such that $\varphi: \widetilde{F} \rightarrow F$ is locally an isometry. Let $d: \widetilde{F} \rightarrow \mathbb{R}$ denote the oriented distance to the straight line $\widetilde{g}{ }^{2}$ We claim that the homotopy

$$
H_{t}:[0,1] \rightarrow F \times \mathbb{R}, \quad s \mapsto\left(h_{t}(s), d\left(\tilde{h}_{t}(s)\right)\right),
$$

which projects to the homotopy $h_{t}$ on $F$, is an isotopy. This claim implies that $H_{0}:[0,1] \rightarrow F \times \mathbb{R}$ is an unknot $O$ that projects to $h_{0}=c$; therefore, it finishes the proof.

In order to prove that $H_{t}$ is an isotopy, we assume towards a contradiction that $H_{t}$ is not injective for some fixed $t$. Without loss of generality we assume $t=0$, ie $\tilde{h}_{t}=\tilde{c}$. If there exist $s \neq r \in[0,1)$, such that $H_{0}(s)=H_{0}(r)$, then, by definition of $H_{0}$, the points $\tilde{p_{1}}=\widetilde{c}(s)$ and $\tilde{p_{2}}=\tilde{c}(r)$ in $\widetilde{F}$ satisfy

$$
\varphi\left(\tilde{p_{1}}\right)=\varphi\left(\tilde{p_{2}}\right), \quad d\left(\tilde{p_{1}}\right)=d\left(\tilde{p_{2}}\right)
$$

As $d\left(\tilde{p_{1}}\right)=d\left(\tilde{p_{2}}\right)$, there is a geodesic segment parallel to $\tilde{g}$ from $\tilde{p_{1}}$ to $\tilde{p_{2}}$. The length of this segment is an integer $k$ since $\varphi\left(\tilde{p_{1}}\right)=\varphi\left(\tilde{p_{2}}\right)$. It follows that $\tilde{p_{2}}=\tilde{c}(k+s)$ if the sign of $k$ is chosen correctly. This is seen by lifting $c$ to $\widetilde{F}$ such that the lift starts at $\tilde{g}(k)=\tilde{c}(k)$; see Figure 3 for a case with $k=1$.

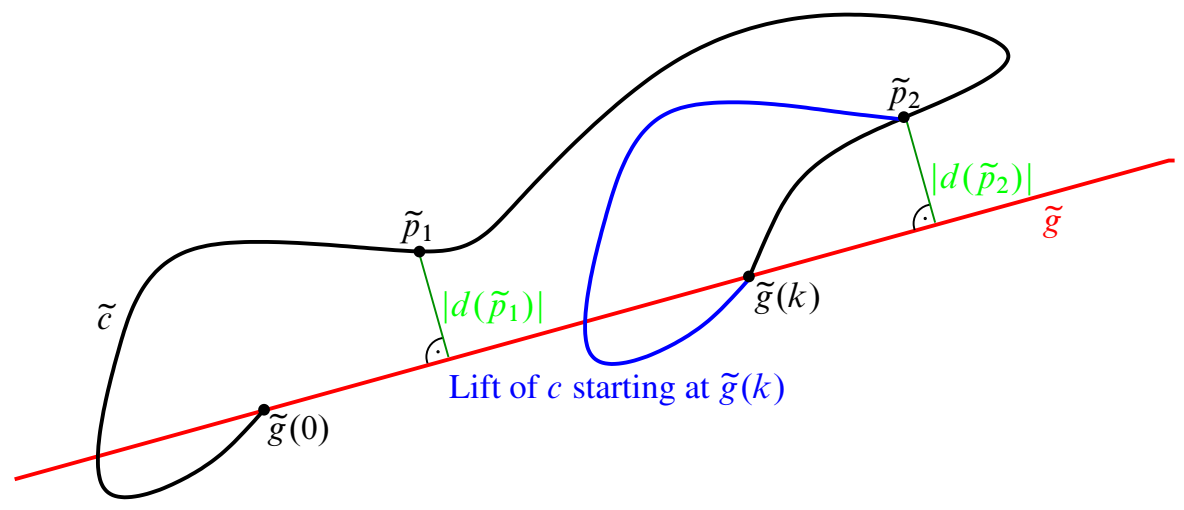

Figure 3: The curve $\left.\widetilde{c}\right|_{[0,1]}$ (black) intersects $\left.\widetilde{c}\right|_{[k, k+s]}$ (blue) in $\tilde{p}_{2}$.

But $\widetilde{c}(r)=\widetilde{c}(k+s)$ and $k+s \neq r$ contradict the injectivity of $\tilde{h}_{t}=\widetilde{c}$.

\footnotetext{
${ }^{2}$ Ordinary Euclidean distance of points in $\widetilde{F}=\mathbb{R}^{2}$ to the straight line $\tilde{g}$ with a sign depending on whether the point is on the left or the right of $\tilde{g}$.
} 
Let us shortly introduce notation and the general strategy for the proof of Theorem 2. In the following $S^{1} \times S^{1}$ denotes the standard torus in $\mathbb{R}^{3}$ and $N\left(S^{1} \times S^{1}\right)$ a tubular neighborhood of $S^{1} \times S^{1}$. Also, we denote the curve obtained by projecting a knot $K$ in $N\left(S^{1} \times S^{1}\right)$ to $S^{1} \times S^{1}$ by $\pi(K)$. Such a curve $\pi(K)$ (together with crossing information) provides a knot diagram on $S^{1} \times S^{1}$ for the knot $K$ in $N\left(S^{1} \times S^{1}\right) \cong$ $S^{1} \times S^{1} \times \mathbb{R}$.

To show the adjacency $K_{1} \leq_{g} K_{2}$ for the knots $K_{2}=T(a, b)$ and $K_{1}=T(n, m)$, ie to show that $d_{g}\left(K_{2}, K_{1}\right)$ is less than or equal (and thus equal) to $u\left(K_{2}\right)-u\left(K_{1}\right)$, we proceed as follows. We isotope $K_{2}$ and $K_{1}$ into $N\left(S^{1} \times S^{1}\right)$ in such a way that:

(I) $\pi\left(K_{1}\right)$ is simple closed (thus, $K_{1}$ is unknotted in $\left.N\left(S^{1} \times S^{1}\right)\right)$.

(II) $K_{2}$ is homotopic to $K_{1}$ in $N\left(S^{1} \times S^{1}\right)$.

(III) $\pi\left(K_{2}\right)$ has $2\left(u\left(K_{2}\right)-u\left(K_{1}\right)\right)$ crossings.

In all our cases $\pi\left(K_{2}\right)$ will have an injective lift to the universal cover $\mathbb{R}^{2}$. This together with (I) and (II) yields that $\pi\left(K_{2}\right)$ is a presimple curve in $S^{1} \times S^{1}$. Thus, Remark 8 applies and, because of (III), guaranties the existence of $u\left(K_{2}\right)-u\left(K_{1}\right)$ crossing changes in $N\left(S^{1} \times S^{1}\right) \cong S^{1} \times S^{1} \times \mathbb{R}$ changing $K_{2}$ to the unknot. This unknot is homotopic to $K_{1}$ by (II) and thus isotopic to $K_{1}$ by Remark 6 .

Before giving a proof of Theorem 2, we apply this strategy in a concrete example.

Example 9 In this example, we will show that $T(3,5)$ is Gordian adjacent to $T(3,7)$. Since $u(T(3,7))-u(T(3,5))=2$ we need to show that we can change $T(3,7)$ to $T(3,5)$ via 2 crossing changes. First we isotope $T(3,7)$ into $N\left(S^{1} \times S^{1}\right)$ as shown on the left in Figure 4.

Projecting this $T(3,7)$ to $S^{1} \times S^{1}$ yields a curve $\pi(T(3,7))$ with 4 crossings that is presimple; namely, $\pi(T(3,7))$ has an injective lift to $\mathbb{R}^{2}$ and is homotopic to the standard embedding of the torus knot $T(3,5)$. Thus, by Remark 8 changing 2 of the crossings suffices to produce a knot $K$ in $N\left(S^{1} \times S^{1}\right)$ that is unknotted. As the knot $K$ and the standard $T(3,5)$ are homotopic unknots in $N\left(S^{1} \times S^{1}\right)$ they are isotopic in $N\left(S^{1} \times S^{1}\right)$ by Remark 6. In particular, $K$ and $T(3,5)$ are isotopic as knots in $\mathbb{R}^{3}$; thus, $d_{g}(T(3,5), T(3,7))=2$. In this example with only 4 crossings one can quickly exhibit the knot $K$ explicitly. For example, the right side of Figure 4 provides a knot $K$ that is obtained from the knot on the left side of Figure 4 by performing two crossing changes in $N\left(S^{1} \times S^{1}\right)$ and that is isotopic to the standard $T(3,5)$ as predicted by Remark 8 . This last isotopy can be seen by applying braid relations (similarly as in the proof of Proposition 5) and checking that these can be realized while staying within $N\left(S^{1} \times S^{1}\right)$. 

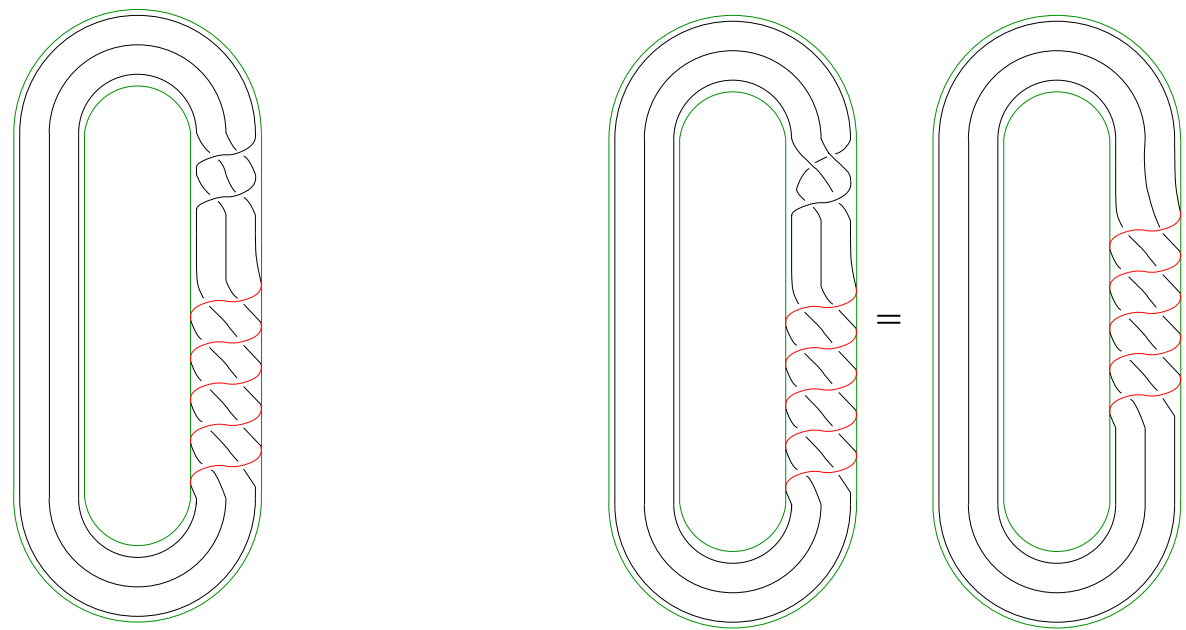

Figure 4: Knots contained in a tubular neighborhood of the standard torus (green) that are homotopic in this neighborhood. Five arcs (red) are on the upper half of the torus, the rest of the knots (black) lie on the lower half. Left: The knot $T(3,7)$ with 4 crossings when projected on to the torus. Right: Two isotopic (in a neighborhood of the torus) occurrences of the knot $T(3,5)$, one of them without crossings.

Proof of Theorem 2 By assumption $(a, b)$ and $(n, m)$ are pairs of coprime natural numbers such that $n \leq a$ and $m \leq b$. Without loss of generality we suppose that $a<b$ and $n<m$.

Let us first consider the case $n=a$, for which we proceed as in Example 9. We need to show that $d_{g}(T(a, b), T(n, m))$ is equal to

$$
u(T(a, b))-u(T(n, m))=\frac{(b-1)(a-1)}{2}-\frac{(m-1)(n-1)}{2}=\frac{(b-m)(a-1)}{2} .
$$

We consider the knot $T(a, b)$ as the closure of the braid $\left(\sigma_{1} \sigma_{2} \cdots \sigma_{a-1}\right)^{b}$ and isotope it into a neighborhood $N\left(S^{1} \times S^{1}\right)$ of the standard torus $S^{1} \times S^{1}$ in $\mathbb{R}^{3}$. Namely, we isotope $m$ arcs on the upper half of the torus and the rest of $T(a, b)$ on the lower half of the torus, in such a way that the curve $\pi(T(a, b))$ winds $m$ times around the core of $S^{1} \times S^{1}$ and $n=a$ times in the direction of the core of $S^{1} \times S^{1}$; see left-hand side of Figure 4. Since $n$ and $m$ are coprime, there is a simple closed curve in $S^{1} \times S^{1}$ that is homotopic to $\pi(T(a, b))$ by the second part of Remark 6 , namely the standard embedding of the torus knot $T(n, m)$ in $S^{1} \times S^{1}$. Also, $\pi(T(a, b))$ lifts injectively to the universal cover $\mathbb{R}^{2}$; thus, $\pi(T(a, b))$ is presimple. The $m$ arcs do not intersect the rest of the curve $\pi(T(a, b))$ on the torus, so $\pi(T(a, b))$ 
has $(b-m)(a-1)$ crossings on the torus. By Remark 8 we need to change at most $(b-m)(a-1) / 2$ crossings in the diagram on the torus (which correspond to crossing changes in $\left.N\left(S^{1} \times S^{1}\right) \cong S^{1} \times S^{1} \times \mathbb{R}\right)$ to get an unknot $K$ in $N\left(S^{1} \times S^{1}\right)$. As the unknotted $K$ and the standard $T(n, m)$ are homotopic in $N\left(S^{1} \times S^{1}\right)$ they are also isotopic by Remark 6 . Of course $K$ is isotopic to $T(n, m)$ in $\mathbb{R}^{3}$ via the same isotopy as in $N\left(S^{1} \times S^{1}\right)$. Therefore, $d_{g}(T(a, b), T(n, m)) \leq(b-m)(a-1) / 2$ as we wanted. The same argument works if $m=b$ or $a=m$.

This leaves the case $n<a$ and $m<b$. In the first case we interpreted $T(a, b)$ as the closure of a braid on $a$ strands, in the following we see $T(a, b)=T(b, a)$ as a braid on $b$ strands. We may assume $m>b-a$, otherwise we replace (inductively) $a, b$ by $a, b-a$ (respectively by $b-a, a$ if $b-a<a$ ) since by the first case $T(a, b-a) \leq_{g} T(a, b)$. To apply the same idea as before we reduce the braid on $b$ strands to one on $m$ strands. More precisely, the representation of $T(a, b)$ as the closure of the $b$-strand braid

$$
\left(\sigma_{1} \cdots \sigma_{b-1}\right)^{a}=\sigma_{a} \cdots \sigma_{1}\left(\sigma_{2} \cdots \sigma_{b-1}\right)^{a},
$$

has the same closure as the $(b-1)$-strand braid

$$
\tau_{b-1}=\sigma_{a-1} \cdots \sigma_{1}\left(\sigma_{1} \cdots \sigma_{b-2}\right)^{a}
$$

see Figure 5.
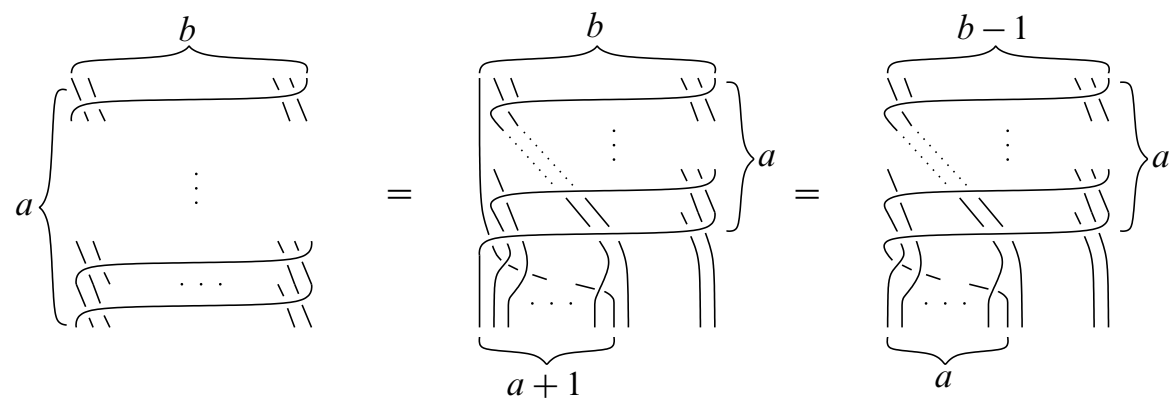

Figure 5: The first equality is the pictorial version of (3). The second equality is a Markov destabilization, ie a Reidemeister I move on the closed braid.

If $m=b-1$, we isotope $T(a, b)$ (seen as the closure of $\left.\tau_{b-1}\right)$ into $N\left(S^{1} \times S^{1}\right)$ such that $n$ of the $a$ over-passing arcs in the right part of Figure 5 project to the upper half of the torus and the rest of $\pi(T(a, b))$, including $a-1+(a-n)(b-2)$ crossings, lies on the lower half. The curve $\pi(T(a, b))$ is presimple since it winds $n$ respectively $m$ times around the torus, ie it is homotopic in $N\left(S^{1} \times S^{1}\right)$ to the standard embedding of the knot $T(n, m)$, and $\pi(T(a, b))$ lifts injectively to $\mathbb{R}^{2}$. Therefore, we 
can use Remark 8 to get $T(n, m)$ by at most $\frac{a-1+(a-n)(b-2)}{2}$ crossing changes. Thus, $d_{g}(T(n, m), T(a, b))$ is less than or equal to

$\frac{a-1+(a-n)(b-2)}{2}=\frac{(a-1)(b-1)}{2}-\frac{(n-1)(b-2)}{2}=u(T(a, b))-u(T(n, m))$.

Suppose now $m<b-1$. We no longer isotope $T(a, b)$ into $N\left(S^{1} \times S^{1}\right)$. We first apply some crossing changes in $\mathbb{R}^{3}$ and then isotope the result into $N\left(S^{1} \times S^{1}\right)$. Namely, we change a crossing in $\tau_{b-1}$ to get

$$
\sigma_{a-1} \cdots \sigma_{2} \sigma_{1}^{-1}\left(\sigma_{1} \cdots \sigma_{b-2}\right)^{a}=\sigma_{a-1} \cdots \sigma_{2} \sigma_{2} \cdots \sigma_{b-2}\left(\sigma_{1} \cdots \sigma_{b-2}\right)^{a-1}
$$

and then replace in (4) the part $\left(\sigma_{1} \cdots \sigma_{b-2}\right)^{a-1}$ by $\sigma_{a-1} \cdots \sigma_{1}\left(\sigma_{2} \cdots \sigma_{b-2}\right)^{a-1}$ as in (3), which has the same closure as the $b-2$ braid

$$
\tau_{b-2}=\left(\sigma_{a-2} \cdots \sigma_{1} \sigma_{1} \cdots \sigma_{b-3}\right)^{2}\left(\sigma_{1} \cdots \sigma_{b-3}\right)^{a-2}
$$

see Figure 6.
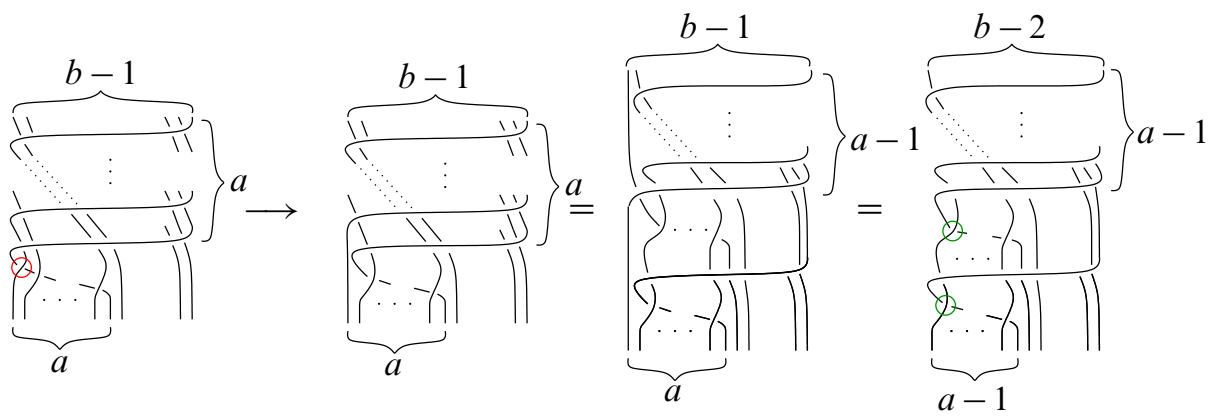

Figure 6: The arrow $\longrightarrow$ indicates the changing of the marked (red) crossing. The two equalities are seen as in Figure 5. The two marked (green) crossings on the right side indicate the crossing changes that are necessary to obtain $\tau_{b-3}$ from $\tau_{b-2}$, which is needed when $m<b-2$.

If $m=b-2$ we isotope the closure of $\tau_{b-2}$ into $N\left(S^{1} \times S^{1}\right)$ in such away that it homotopic to $T(n, m)$; namely, such that $n$ of the $a$ over-passing arcs get to lie on the upper part of the torus and the reminding part (including $2(a-2)+(a-n)(b-3)$ crossings) lies on the lower part. Therefore, Remark 8 implies that $T(n, m)$ can be obtained from the closure of $\tau_{b-2}$ by changing $\frac{2(a-2)+(a-n)(b-3)}{2}$ crossings. Thus, 
$d_{g}(T(n, m), T(a, b))$ is less than or equal to

$$
\begin{aligned}
1+\frac{2(a-2)+(a-n)(b-3)}{2} & =\frac{2 a-2+(a-n)(b-3)}{2} \\
& =\frac{2 a-2+(a-1)(b-3)}{2}-\frac{(n-1)(b-3)}{2} \\
& =\frac{(a-1)(b-1)}{2}-\frac{(n-1)(b-3)}{2} \\
& =u(T(a, b))-u(T(n, m)) .
\end{aligned}
$$

For general $m>b-a$ it follows similarly that we need to change

$$
1+2+\cdots+(b-m-1)=\frac{(b-m)(b-m-1)}{2}
$$

crossings of $T(a, b)$ to get the closure of the $m$ braid

$$
\tau_{m}=\left(\sigma_{a-(b-m)} \cdots \sigma_{1} \sigma_{1} \cdots \sigma_{m-1}\right)^{b-m}\left(\sigma_{1} \cdots \sigma_{m-1}\right)^{a-(b-m)} ;
$$

see Figure 7. In Figure 6 we have indicated (green) how to obtain $\tau_{b-3}$ from $\tau_{b-2}$.

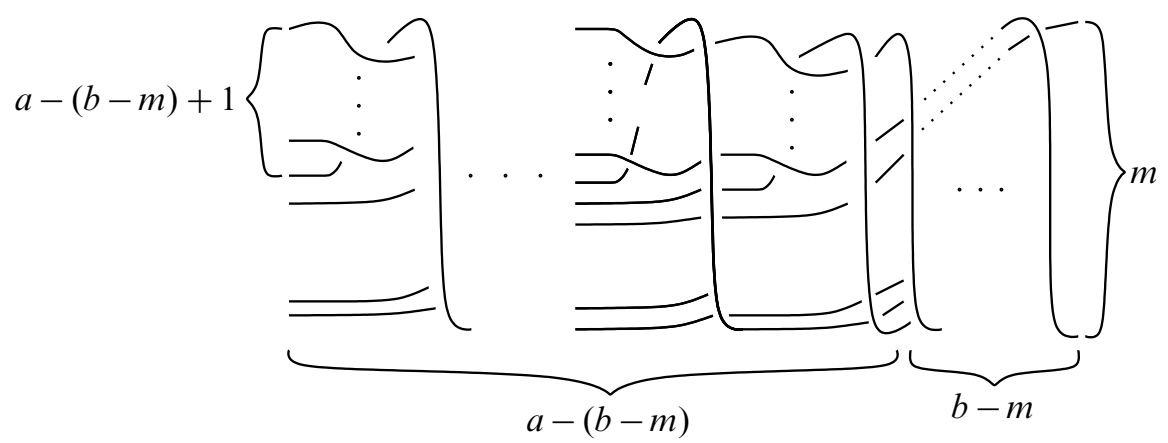

Figure 7: The braid $\tau_{m}$, which can be obtained from $T(a, b)$ by $1+2+$ $\cdots+(b-m-1)$ crossing changes.

We isotope the closure of $\tau_{m}$ into $N\left(S^{1} \times S^{1}\right)$ such that $n$ of the $a$ over-passing arcs lie on the upper half of the torus and $(b-m)(a-(b-m))+(a-n)(m-1)$ crossings on the lower half. Thus, we get $T(n, m)$ from $\tau_{m}$ by changing $\frac{(b-m)(a-(b-m))+(a-n)(m-1)}{2}$ crossings by Remark 8 . Combined we have that $d_{g}(T(n, m), T(a, b))$ is less than or equal to

$$
\frac{(b-m)(b-m-1)+(b-m)(a-(b-m))+(a-n)(m-1)}{2},
$$

which is equal to $u(T(a, b))-u(T(n, m))$. 


\section{Levine-Tristram signatures as obstructions to adjacency}

The goal here is to prove Theorem 3 using Levine-Tristram signatures [14; 23]. For torus knots they are easy to calculate and yield good obstructions to adjacency; see Lemma 15 and Proposition 13, respectively.

Definition $10 \quad[14 ; 23]$ Let $A$ be a Seifert matrix of a knot $K$ and $\omega$ in $S^{1} \backslash\{1\} \subset \mathbb{C}$. The $\omega$-signature $\sigma_{\omega}(K) \in \mathbb{Z}$ is defined to be the number of negative eigenvalues minus the number of positive eigenvalues of the Hermitian matrix $(1-\omega) A+(1-\bar{\omega}) A^{t}$.

The $\omega$-signature is independent of the choice of Seifert matrix and $\sigma_{\omega}=\sigma_{\bar{\omega}}$. Setting $\omega=-1$ one recovers the classical signature $\sigma_{-1}$ introduced by Trotter [24]. Note that our nonstandard sign convention of signatures is such that all (positive) torus knots have positive signature, eg $\sigma_{-1}(T(2,3))=2$ rather than $\sigma_{-1}(T(2,3))=-2$.

We say $\omega$ in $S^{1} \backslash\{1\}$ is regular for a knot $K$ if $\omega$ is not a root of the Alexander polynomial of $K$. Regular $\omega$ 's are all $\omega$ for which $(1-\omega) A+(1-\bar{\omega}) A^{t}$ is an invertible matrix, where $A$ is a Seifert matrix for $K$. If $\omega$ is regular for a knot $K$, then the signature $\sigma_{\omega}(K)$ is even, and if $\omega$ is a root of unity of prime order, then $\omega$ is regular for every knot [23]. For a fixed knot $K$ the signature $\sigma_{\omega}(K)$ is piecewise-constant in $\omega$, "jumping" at the nonregular $\omega$ [14].

Let us denote by $s(K)$ the Rasmussen invariant of a knot $K$ [19]. The next lemma shows how $\omega$-signatures and $s$ behave with respect to crossing changes.

Lemma 11 If $K_{-}$is obtained from $K_{+}$via one positive-to-negative crossing change and if $\omega$ is regular for $K_{1}$ and $K_{2}$, then

$$
\sigma_{\omega}\left(K_{-}\right) \in\left\{\sigma_{\omega}\left(K_{+}\right), \sigma_{\omega}\left(K_{+}\right)-2\right\} .
$$

The same holds for the Rasmussen invariant.

Rasmussen used an observation by Livingston [15, Corollary 2 and 3] to prove Lemma 11 for $s$ [19]. For $\omega$-signatures we only found proofs of the following weaker statement in the literature (see Kawauchi [11, Theorem 11.2.1] and [8]).

Corollary 12 Let $K_{1}, K_{2}$ be knots and let $\omega$ be regular for $K_{1}$ and $K_{2}$. Then

$$
\left|\frac{\sigma_{\omega}\left(K_{2}\right)-\sigma_{\omega}\left(K_{1}\right)}{2}\right| \leq d_{g}\left(K_{1}, K_{2}\right) \text {. }
$$

In particular, if $K_{1}$ is adjacent to $K_{2}$, then $\left|\frac{\sigma_{\omega}\left(K_{2}\right)-\sigma_{\omega}\left(K_{1}\right)}{2}\right| \leq u\left(K_{2}\right)-u\left(K_{1}\right)$. 
We provide a proof of Lemma 11 at the end of this section using a variation of Livingston's observation.

As a consequence of Corollary 12 we prove that most torus knots are not adjacent to torus knots of index two as claimed in Remark 4. For index two torus knots the signature equals twice the unknotting number, that is

$$
\frac{\sigma_{-1}(T(2, n))}{2}=u(T(2, n))=\frac{n-1}{2} .
$$

This is also true for $T(3,4)$ and $T(3,5)$, but for all other torus knots $T$ there is a signature defect, ie $u(T)>\frac{\sigma_{-1}(T)}{2}$. Thus, by Corollary 12 ,

$$
d_{g}(T(2, n), T) \geq \frac{\sigma_{-1}(T(2, n))}{2}-\frac{\sigma_{-1}(T)}{2}>u(T(2, n))-u(T)
$$

for all torus knots $T$ not equal to $T(3,4), T(3,5)$ or some $T(2, m)$.

The following proposition explains how Lemma 11 gives another obstruction to Gordian adjacency of torus knots, which is often better than Corollary 12.

Proposition 13 Let $T_{1} \leq_{g} T_{2}$ be a Gordian adjacency of torus knots and let $\omega$ be regular for $T_{1}$ and $T_{2}$. Then $\sigma_{\omega}\left(T_{1}\right) \leq \sigma_{\omega}\left(T_{2}\right)$.

Proof For all torus knots $T$ we have $\frac{s(T)}{2}=u(T)$ [19]. Thus, Lemma 11 yields that an unknotting sequence of any torus knot involves only positive-to-negative crossing changes since $s$ has to drop by 2 with every crossing change. Choose an $\widetilde{\omega}$ that is regular for every knot and such that $\sigma_{\omega}\left(T_{1}\right)=\sigma_{\widetilde{\omega}}\left(T_{1}\right)$ and $\sigma_{\omega}\left(T_{2}\right)=\sigma_{\widetilde{\omega}}\left(T_{2}\right)$. This is for example achieved by a root of unity of prime order that is close to $\omega$. Let now

$$
T_{2}=K_{u\left(T_{2}\right)} \rightarrow K_{u\left(T_{2}\right)-1} \rightarrow \cdots \rightarrow T_{1} \rightarrow \cdots \rightarrow K_{1} \rightarrow K_{0}=O
$$

be an unknotting sequence for $T_{2}$ that contains $T_{1}$. As it involves only positive-tonegative crossing changes we have

$$
\sigma_{\widetilde{\omega}}\left(T_{2}\right) \geq \sigma_{\widetilde{\omega}}\left(K_{u\left(T_{2}\right)-1}\right) \geq \cdots \geq \sigma_{\widetilde{\omega}}\left(T_{1}\right) \geq \cdots \geq \sigma_{\widetilde{\omega}}(O)=0
$$

by Lemma 11 . Therefore $\sigma_{\omega}\left(T_{1}\right)=\sigma_{\widetilde{\omega}}\left(T_{1}\right) \leq \sigma_{\widetilde{\omega}}\left(T_{2}\right)=\sigma_{\omega}\left(T_{2}\right)$.

Remark 14 By the above proof Proposition 13 remains true for any knot $K$ with $\frac{s(K)}{2}=u(K)$. For example, all knots that are closures of positive braids, which include algebraic knots.

We prove Theorem 3 using Proposition 13 and the following combinatorial formula for the Levine-Tristram signatures of torus knots, see [8], for $\sigma_{-1}$ it is originally due to Brieskorn and Hirzebruch $[5 ; 10]$. We denote the cardinality of a finite set $S$ by $\sharp S$. 
Lemma 15 Let $n \geq 2$ and $m \geq 2$ be coprime natural numbers. Set $S=\left\{\frac{k}{n}+\frac{l}{m} \mid\right.$ $1 \leq k \leq n-1,1 \leq l \leq m-1\} \subset[0,2]$. Then for $\theta \in[0,1]$ we have

$$
\sigma_{e^{2 \pi i \theta}}(T(n, m))=\sharp(S \cap[\theta, \theta+1])-\sharp(S \backslash(\theta, \theta+1)) .
$$

Proof of Theorem 3 Fix $n=2 k+1$ and note that $m=\left\lfloor\frac{3}{2} k+1\right\rfloor$ is minimal with $n \leq \frac{4}{3} m+\frac{1}{3}$. By Proposition 5 we have $T(2,2 k+1) \leq g T\left(3,\left\lfloor\frac{3}{2} k+1\right\rfloor\right)$. Together with an easy instance of Theorem $2, T\left(3,\left\lfloor\frac{3}{2} k+1\right\rfloor\right) \leq_{g} T(3, m)$ for all $m \geq\left\lfloor\frac{3}{2} k+1\right\rfloor$, we conclude that $T(2,2 k+1) \leq g T(3, m)$ for all $m \geq\left\lfloor\frac{3}{2} k+1\right\rfloor$.

For the other direction we let $n=2 k+1$ be any odd number and write $m=\left\lceil\frac{3}{2} k-1\right\rceil$, which is the largest $m$ that does not satisfy $n \leq \frac{4}{3} m+\frac{1}{3}$. Thus, we have to show that $T(2,2 k+1) \not \bigsqcup_{g} T(3, m)$. For $k \leq 4$ calculating unknotting numbers yields $T(2,5) \not \mathbb{g}_{g}$ $T(3,2), T(2,7) \not \mathbb{g}_{g} T(3,4)$, and $T(2,9) \not \bigsqcup_{g} T(3,5)$. If $k \geq 5$ we distinguish two cases. Either, $k$ equals 1 or 2 modulo 4 , or $k$ equals 3 or 4 modulo 4 .

For $k=1+4 l, 2+4 l, l \geq 1$ a calculation using Gordon, Litherland and Murasugi's reduction formula [9, Theorem 5.2] shows that

$$
\sigma_{-1}(T(3, m))=2 k-2,
$$

which is strictly less than

$$
\sigma_{-1}(T(2,2 k+1))=2 k \text {. }
$$

Thus, Proposition 13 yields $T(2,2 k+1) \not \bigsqcup_{g} T(3, m)$.

For $k=3+4 l, 4+4 l, l \geq 1$ we use [9, Theorem 5.2] similarly to see that

$$
\sigma_{-1}(T(3, m))=2 k=\sigma_{-1}(T(2,2 k+1)) .
$$

Here $\sigma_{-1}$ does not suffice as obstruction directly, but we use (5) to calculate $\sigma_{\omega}(T(3, m))$ for $\omega$ close to -1 , which yields the desired obstruction. More precisely set

$$
\omega=e^{2 \pi i \theta}, \quad \text { with } \begin{cases}\theta \in\left(\frac{1}{2}-\frac{2}{3 m}, \frac{1}{2}-\frac{1}{3 m}\right) & \text { for } m \text { even, ie } k=3+4 l, \\ \theta \in\left(\frac{1}{2}-\frac{3}{6 m}, \frac{1}{2}-\frac{1}{6 m}\right) & \text { for } m \text { odd, ie } k=4+4 l\end{cases}
$$

By Lemma 15 the value of $\sigma_{\omega}(T(3, m))$ is the same for all these $\omega$.

Claim 16 For all $k=3+4 l, 4+4 l$ with $l \geq 1$ and $\omega$ as in (6), we have $\sigma_{\omega}(T(3, m))=$ $\sigma_{-1}(T(3, m))-2$. 
As the above $\omega$ can be chosen such that $\sigma_{-1}(T(2,2 k+1))=\sigma_{\omega}(T(2,2 k+1))$, Claim 16 and (5) yield

$$
\begin{aligned}
\sigma_{\omega}(T(3, m)) & =\sigma_{-1}(T(3, m))-2<\sigma_{-1}(T(3, m)) \\
& =\sigma_{-1}(T(2,2 k+1))=\sigma_{\omega}(T(2,2 k+1)) .
\end{aligned}
$$

Therefore, $T(2,2 k+1) \not \leq g T(3, m)$ by Proposition 13. It remains to prove Claim 16 .

For the case when $m$ is even, Lemma 15 applied to the knot

$$
T=T(3, m)=T\left(3,\left\lceil\frac{3}{2}(3+4 l)-1\right\rceil\right)=T(3,4+6 l)
$$

yields

$$
\begin{aligned}
\sigma_{-1}(T) & =\sharp\left(S \cap\left[\frac{1}{2}-\frac{1}{3 m}+\varepsilon, \frac{3}{2}-\frac{1}{3 m}+\varepsilon\right]\right)-\sharp\left(S \backslash\left(\frac{1}{2}-\frac{1}{3 m}+\varepsilon, \frac{3}{2}-\frac{1}{3 m}+\varepsilon\right)\right), \\
\sigma_{\omega}(T) & =\sharp\left(S \cap\left[\frac{1}{2}-\frac{1}{3 m}-\varepsilon, \frac{3}{2}-\frac{1}{3 m}-\varepsilon\right]\right)-\sharp\left(S \backslash\left(\frac{1}{2}-\frac{1}{3 m}-\varepsilon, \frac{3}{2}-\frac{1}{3 m}-\varepsilon\right)\right),
\end{aligned}
$$

for $\varepsilon$ small enough. Observe that

$$
\begin{gathered}
\frac{3}{2}-\frac{1}{3 m}=\frac{2}{3}+\frac{5 m-2}{6 m}=\frac{2}{3}+\frac{5(4+6 l)-2}{6(4+6 l)}=\frac{2}{3}+\frac{3+5 l}{4+6 l}=\frac{2}{3}+\frac{3+5 l}{m} \in S, \\
\frac{1}{2}-\frac{1}{3 m}=\cdots=\frac{1}{3}+\frac{5 k}{m}+\frac{1}{3} \frac{1}{m} \notin S .
\end{gathered}
$$

This means

$$
\begin{aligned}
& \left(S \cap\left[\frac{1}{2}-\frac{1}{3 m}-\varepsilon, \frac{3}{2}-\frac{1}{3 m}-\varepsilon\right]\right) \dot{\cup}\left\{\frac{3}{2}-\frac{1}{3 m}\right\}=S \cap\left[\frac{1}{2}-\frac{1}{3 m}+\varepsilon, \frac{3}{2}-\frac{1}{3 m}+\varepsilon\right], \\
& S \backslash\left(\frac{1}{2}-\frac{1}{3 m}-\varepsilon, \frac{3}{2}-\frac{1}{3 m}-\varepsilon\right)=\left(S \backslash\left(\frac{1}{2}-\frac{1}{3 m}+\varepsilon, \frac{3}{2}-\frac{1}{3 m}+\varepsilon\right)\right) \dot{\cup}\left\{\frac{3}{2}-\frac{1}{3 m}\right\} .
\end{aligned}
$$

Therefore, $\sigma_{-1}(T)=2+\sigma_{\omega}(T)$.

If $m$ is odd, we have $m=5+6 l$. Similarly to the even case, we get

$$
\frac{3}{2}-\frac{1}{6 m}=\frac{2}{3}+\frac{4+5 l}{m} \in S, \quad \text { but } \frac{1}{2}-\frac{1}{6 m} \notin S .
$$

The rest of the argument is the same.

It remains to prove Lemma 11 . Let $-K$ denote the mirror image of a knot $K$ (with reversed orientation), and let $K_{1} \# K_{2}$ denote the connected sum of two knots $K_{1}$ and $K_{2}$. 
Lemma 17 Let $\tau$ be a integer valued knot invariant satisfying

- $\tau\left(K_{1} \# K_{2}\right)=\tau\left(K_{1}\right)+\tau\left(K_{1}\right)$ and $\tau\left(-K_{1}\right)=-\tau\left(K_{1}\right)$ for all knots $K_{1}$ and $K_{2}$,

- $\tau(K) \leq g_{s}(K)$ for all knots $K$,

- there exists a knot $K$ with $\tau(K)=1$ that can be transformed to the unknot $O$ by a positive-to-negative crossing change.

Then $\tau$ is a concordance invariant, $|\tau(K)| \leq g_{s}(K)$ for all knots $K$ and

$$
0 \leq \tau\left(K_{+}\right)-\tau\left(K_{-}\right) \leq 1
$$

whenever $K_{-}$is a knot obtained from $K_{+}$by a positive-to-negative crossing change.

Lemma 17 is a variation of the statement in [15, Corollary 2 and 3]. The first two assertions are given in [15, Corollary 2]. The proof of the third assertion given in [15] needs to be modified as follows to yield a proof Lemma 17. Replace, in the proof of [15, Corollary 3], the knot $T(2,3)$ by a knot $K$ with $\tau(K)=1$ that can be unknotted by changing one positive crossing to a negative one. This is necessary since we do not assume that $\tau(T(2,3))=1$.

Proof of Lemma 11 Rasmussen proves all conditions of Lemma 17 for $\tau=\frac{s}{2}$ in [19] (note that $\frac{s(T(2,3))}{2}=1$ ). This yields the desired result for $s$. For every $\omega$ that is regular for $K_{-}$and $K_{+}$, there is a root of unity $\widetilde{\omega}$ of prime order with $\sigma_{\widetilde{\omega}}\left(K_{-}\right)=\sigma_{\omega}\left(K_{-}\right)$ and $\sigma_{\widetilde{\omega}}\left(K_{+}\right)=\sigma_{\omega}\left(K_{+}\right)$. Thus, it is enough to check the three conditions of Lemma 17 for $\frac{\sigma_{\omega}}{2}$, where $\omega$ is a root of unity of prime order.

For signatures $\sigma_{\omega}\left(K_{1} \# K_{2}\right)=\sigma_{\omega}\left(K_{1}\right)+\sigma_{\omega}\left(K_{2}\right)$ and $\sigma_{\omega}\left(-K_{1}\right)=-\sigma_{\omega}\left(K_{1}\right)$ follow from the fact that $A_{1} \oplus A_{2}$ is a Seifert matrix for $K_{1} \# K_{2}$ and $-A_{1}$ is a Seifert matrix for $-K_{1}$ if $A_{1}$ and $A_{2}$ are Seifert matrices for $K_{1}$ and $K_{2}$, respectively. If $\omega=-1$, the second condition is proven by Murasugi [18] and for the third condition we can choose $K$ to be $T(2,3)$ as $\sigma_{-1}(T(2,3))=2$. In general, fix a root of unity $\omega$ of prime order. The second condition is proven by Tristram [23]. ${ }^{3}$ It remains to find a suitable $K$ for the third condition. For a natural number $k$ let $T(2 k-1)$ be the positive twist knot with $2 k-1$ half-twists. These knots can be unknotted by a positive-to-negative crossing change. One checks that

$$
A=\left[\begin{array}{cc}
-k & 1 \\
0 & -1
\end{array}\right]
$$

\footnotetext{
${ }^{3}$ Note that Tristram formulates this result only for $\omega=-1$ and $\omega=e^{(\pi i(p-1)) / p}$, where $p$ is any odd prime; however, his proof works for every root of unity of prime order.
} 
is a Seifert matrix for $T(2 k-1)$. For sufficiently large $k$ both eigenvalues of the Hermitian matrix $(1-\omega) A+(1-\bar{\omega}) A^{t}$ are negative. Thus, $K$ can be chosen to be $T(2 k-1)$ for a sufficiently large $k$.

\section{A bound on Gordian adjacency for torus knots of higher indices}

This section is concerned with the question, when is $T(a, n) \leq_{g} T(b, m)$ for fixed $a<b$ and $n, m$ large? Concretely we study the numbers

$$
\underline{c}(a, b)=\liminf _{m \rightarrow \infty} \frac{n(m)}{m}, \quad \bar{c}(a, b)=\limsup _{m \rightarrow \infty} \frac{n(m)}{m},
$$

where $n(m)$ denotes the largest integer such that $T(a, n(m)) \leq_{g} T(b, m)$. We suspect, but cannot prove, that $\underline{c}(a, b)=\bar{c}(a, b)$ for all $a<b \in \mathbb{N}$. Certainly we have that $\underline{c}(2,3)=\bar{c}(2,3)=\frac{4}{3}$ by Theorem 3 . Also note that $1 \leq \underline{c}(a, b)$ by Theorem 2 and $\bar{c}(a, b) \leq \frac{b-1}{a-1}$ since

$$
\frac{(a-1)(n(m)-1)}{2}=u(T(a, n(m))) \leq u(T(b, m))=\frac{(b-1)(m-1)}{2} .
$$

Using $\omega$-signatures we get an upper bound for $\bar{c}(a, b)$ that is strictly better than $\frac{b-1}{a-1}$.

Proposition 18 If $a \leq b \in \mathbb{N}$, then

$$
\bar{c}(a, b) \leq \frac{a\left\lceil\frac{b}{a}\right\rceil^{2}-(a+2 b)\left\lceil\frac{b}{a}\right\rceil+b(b+1)}{(a-1) b} \leq \frac{b}{a} .
$$

A calculation shows that $\left(a\left\lceil\frac{b}{a}\right\rceil^{2}-(a+2 b)\left\lceil\frac{b}{a}\right\rceil+b(b+1)\right) /((a-1) b)=\frac{b}{a}$ if and only if $a$ divides $b$. If for example $b-a$ equals 1 , Proposition 18 yields

$$
\bar{c}(a, a+1) \leq \frac{a+2}{a+1} .
$$

This is better than $\frac{b}{a}=\frac{a+1}{a}$ or even $\frac{b-1}{a-1}$, but we only know it to be optimal for $a=2$; namely, $\underline{c}(2,3)=\bar{c}(2,3)=\frac{4}{3}$.

Proof We will use an approximation given by Gambaudo and Ghys in [8, Proposition 5.2]. Let $l$ be a natural number. Then

$$
\left|\sigma_{e^{2 \pi i \theta}}(T(b, m))-m\left(2(b-(2 l-1)) \theta+\frac{2 l(l-1)}{b}\right)\right| \leq 2 b \quad \text { for } \frac{l-1}{b}<\theta \leq \frac{l}{b} .
$$


Proposition 13 yields $\sigma_{\omega}(T(b, m))-\sigma_{\omega}(T(a, n(m))) \geq 0$ if $\omega$ is regular for $T(b, m(n))$ and $T(a, n)$. By the approximation we get

$$
\begin{array}{r}
m\left(2(b-(2 l-1)) \theta+\frac{2 l(l-1)}{b}\right)-n(m)\left(2\left(a-\left(2 l^{\prime}-1\right)\right) \theta+\frac{2 l^{\prime}\left(l^{\prime}-1\right)}{a}\right) \\
\geq-2(a+b),
\end{array}
$$

where $l$ and $l^{\prime}$ are natural numbers with $\frac{l-1}{b}<\theta \leq \frac{l}{b}$ and $\frac{l^{\prime}-1}{a}<\theta \leq \frac{l^{\prime}}{a}$, respectively. Choosing $\theta=\frac{1}{a}$, (7) becomes

$$
m\left(2 \frac{\left(b-\left(2\left\lceil\frac{b}{a}\right\rceil-1\right)\right)}{a}+2 \frac{\left\lceil\frac{b}{a}\right\rceil\left(\left\lceil\frac{b}{a}\right\rceil-1\right)}{b}\right)-n(m) 2 \frac{a-1}{a} \geq-2(a+b)
$$

or equivalently

$$
\frac{n(m)}{m} \leq \frac{a\left\lceil\frac{b}{a}\right\rceil^{2}-(a+2 b)\left\lceil\frac{b}{a}\right\rceil+b(b+1)}{(a-1) b}+\frac{a(a+b)}{m(a-1)} .
$$

This proves the first inequality. ${ }^{4}$ The second inequality can be checked by a short calculation.

Remark 19 Our choice $\theta=\frac{1}{a}$ is the best possible and yields the optimal bound for $\bar{c}(a, b)$ that can be achieved using the properties of signatures from Lemma 11. This can be checked using the above approximation from [8].

In order to determine $\underline{c}(a, b)$ and $\bar{c}(a, b)$ for $(a, b) \neq(2,3)$, we now wish to find geometric constructions in the spirit of Section 2 that at least for some $a$ and $b$ yield a lower bound for $\underline{c}(a, b)$ that is equal to the upper bound given by Proposition 18 . So far we have only found constructions giving lower bounds that do not coincide with the upper bounds, eg $\frac{5}{3} \leq \underline{c}(2,4) \leq \bar{c}(2,4) \leq 2$ and $\frac{9}{8} \leq \underline{c}(3,4) \leq \bar{c}(3,4) \leq \frac{5}{4}$.

\section{Algebraic adjacency}

In this section we compare $\leq_{g}$ with an adjacency notion for plane curve singularities. We first recall the notion of an algebraic knot following Milnor [16]. Let $f:\left(\mathbb{C}^{2}, 0\right) \rightarrow(\mathbb{C}, 0)$ be a polynomial function or a holomorphic function germ that is

\footnotetext{
${ }^{4}$ Note the following technical point. If $\omega=e^{2 \pi i(1 / a)}$ is nonregular for $T(b, m(n))$ or $T(a, n)$, then Lemma 11 cannot be applied as above. Instead one chooses a sequence of $\theta_{k}$ tending to $\frac{1}{a}$, such that every $e^{2 \pi i \theta_{k}}$ is regular for $T(b, m(n))$ and $T(a, n)$. Inequality (8) is then obtained by choosing $\theta=\theta_{k}$ in inequality (7) and letting $k$ tend to infinity.
} 
irreducible ${ }^{5}$ in the ring of holomorphic function germs $\mathbb{C}\{x, y\}$ and has an isolated singularity at the origin. The transversal intersection of its zero set $V(f) \subseteq \mathbb{C}^{2}$ with a sufficiently small sphere around the origin $S_{\varepsilon}^{3}=\left\{(x, y) \in \mathbb{C}^{2} \mid\|x\|^{2}+\|y\|^{2}=\varepsilon^{2}\right\}$ is a knot in $S_{\varepsilon}^{3} \cong S^{3}$ called the knot of the singularity of $f$. For example, the torus knot $T(n, m)$ is the knot of the singularity of $x^{n}-y^{m}$. In this case the small sphere can be taken to be the standard unit sphere $S^{3}$; thus, $T(n, m)=S^{3} \cap\left\{(x, y) \in \mathbb{C}^{2} \mid x^{n}-y^{m}=0\right\} \subset S^{3}$. Knots that can occur as knots of singularities are called algebraic.

Arnold studied adjacency of singular function germs [1, Definition 2.1]; see also Siersma [21]. As we are interested in knots, we study singular function germs only up to topological type, ie up to the isotopy class of their knots of singularity; see eg Brieskorn and Knörrer [6]. Thus, we use the following version of adjacency.

A deformation of $f \in \mathbb{C}\{x, y\}$ is a smooth family $h_{t} \in \mathbb{C}\{x, y\}$, defined for small enough real $t \geq 0$, with $h_{0}=f$.

Definition 20 Let $K_{1}$ and $K_{2}$ be algebraic knots. We say $K_{1}$ is algebraically adjacent to $K_{2}$, denoted by $K_{1} \leq_{a} K_{2}$, if there exists a germ $f \in \mathbb{C}\{x, y\}$ with $K_{2}$ as knot of the singularity and a deformation $h_{t}$ of $f$, such that for small nonzero $t$ the germ $h_{t}$ has $K_{1}$ as knot of the singularity.

Remark 21 Since every holomorphic germ yields the same knot as its Taylor polynomials of large enough degrees, one can study polynomials or holomorphic germs.

Isotopy classes of algebraic knots can be identified canonically with $\mu$-constanthomotopy classes of irreducible germs $\left(\mathbb{C}^{2}, 0\right) \rightarrow(\mathbb{C}, 0)$, where $\mu$ is the Milnor number. With this identification the above notion of adjacency for algebraic knots corresponds to the concept of $\mu$-adjacency studied by Siersma in [21]. We sketch this identification. If two plane curves can be connected by a $\mu$-constant path, then the associated algebraic knots are isotopic; see Tráng and Ramanujam [22]. For the converse assume that two irreducible germs $f_{0}$ and $f_{1}$ have the same knot of singularity. After coordinate changes they are both of the form $y^{m}+c_{m-1}(x) y^{m-1}+\cdots+c_{0}(x)$, where $m$ is the multiplicity of $f_{0}$ and $f_{1}$, and where the $c_{k} \in \mathbb{C}\{x\}$ are holomorphic germs with $c_{k}(0)=0$. If $f_{0}$ and $f_{1}$ have the same knot of singularity, then they have the same essential terms in their corresponding Puiseux expansions $y_{0}\left(x^{1 / m}\right)$ and $y_{1}\left(x^{1 / m}\right)$; see eg [6]. Thus, the two Puiseux expansions can be connected by a family of Puiseux expansions $y_{t}\left(x^{1 / m}\right)$ with the same essential terms. This yields a

\footnotetext{
${ }^{5}$ With the weaker assumption 'squarefree' most of what is done in this section still works, but we get links instead of knots.
} 
$\mu$-constant family of germs

$$
f_{t}=\prod_{\xi^{m}=1}\left(y-y_{t}\left(\xi x^{1 / m}\right)\right) \in \mathbb{C}\{x, y\}
$$

that connects $f_{0}$ to $f_{1}$.

As described in the introduction both notions of adjacency have a lot of properties in common. For example, Theorem 2 is known and easy to show for $\leq_{a}$ instead of $\leq_{g}$.

Proposition 22 If $n \leq a$ and $m \leq b$, then $T(n, m) \leq_{a} T(a, b)$.

Proof Regard $T(a, b)$ as the knot of the singularity of $y^{a}-x^{b}$ and choose as deformation $h_{t}(x, y)=y^{a}-x^{b}+t\left(y^{n}-x^{m}\right)$. For $t$ small (but fix) we make, in a small chart around the origin, a biholomorphic coordinate change, which does not change the topological type of the singularity, such that $h_{t}=y^{n}\left(t+y^{a-n}\right)-x^{m}\left(t+x^{b-m}\right)$ becomes $y^{n}-x^{m}$.

The obstruction to Gordian adjacency given in Corollary 12 also holds for algebraic adjacency. Actually Corollary 12 and its counterpart for $\leq_{a}$ are a consequence of the fact that $\left|\left(\sigma_{\omega}\left(K_{2}\right)-\sigma_{\omega}\left(K_{1}\right)\right) / 2\right|$ is less than or equal to the cobordism distance of $K_{1}$ and $K_{2}$, and the following. For algebraic knots both $K_{1} \leq g K_{2}$ and $K_{1} \leq{ }_{a} K_{2}$ yield a cobordism in $S^{3} \times[0,1]$ between $K_{1}$ and $K_{2}$ of minimal genus $u\left(K_{2}\right)-u\left(K_{1}\right)=$ $g_{s}\left(K_{2}\right)-g_{s}\left(K_{1}\right)$. For an algebraic adjacency given by a deformation $h_{t}$ this cobordism is given as follows. Let $S_{2}$ be a sufficiently small sphere with $K_{2}=S_{2} \cap V\left(h_{0}\right)$. Then, by transversality, $t$ can be chosen small enough such that $S_{2} \cap V\left(h_{t}\right)$ is still $K_{2}$ and $K_{1}=S_{1} \cap V\left(h_{t}\right)$ for a small enough sphere $S_{1}$. By a small perturbation of $h_{t}$ the zero set $V\left(h_{t}\right)$ becomes a smooth algebraic curve $F$ with $K_{2}=S_{2} \cap F$ and $K_{1}=S_{1} \cap F$. The cobordism between $K_{1}$ and $K_{2}$, which is given by $F$, has minimal genus $u\left(K_{2}\right)-u\left(K_{1}\right)=g_{s}\left(K_{2}\right)-g_{s}\left(K_{1}\right)$ by the Thom conjecture [13, Corollary 1.3].

Despite these similarities the two notions of adjacency do not agree for algebraic knots or even torus knots. The obstruction given in Proposition 13, that the signature decreases on Gordian adjacent torus knots, does not hold for algebraic adjacency. Concretely we have $T(2,15) \leq_{a} T(3,10)$, but $T(2,15) \not \leq_{g} T(3,10)$ by Theorem 3 . We generalize an example of an algebraic adjacency calculated by Arnold [1, $\left.A_{5} \leftarrow E_{6}\right]$. This gives a large class of examples of algebraic adjacencies of torus knots, including $T(2,15) \leq_{a} T(3,10)$, which are not covered by Proposition 22.

Proposition 23 Let $a, b, c$ be natural numbers with $a \leq b$, then $T(a, b c) \leq{ }_{a} T(b, a c)$. In particular, $T(2,3 c) \leq{ }_{a} T(3,2 c)$. 
Proof Suppose that $a<b$, regard $T(b, a c)$ as the knot of the singularity of $y^{b}-x^{a c}$ and choose $h_{t}=y^{b}-\left(x^{c}-t y\right)^{a}$ as deformation. For fixed small $t>0$ we change coordinates by $x \mapsto x, y \mapsto \frac{x^{c}-y}{t}$, so $h_{t}=\left(\frac{x^{c}-y}{t}\right)^{b}-y^{a}$. Then we write $h_{t}=t^{-b} x^{b c}-$ $y^{a}+p(x, y)$, where the degrees of the monomials in $p$ lie on the line through $(b c, 0)$ and $(0, b)$; in particular, they lie strictly above the line through $(b c, 0)$ and $(0, a)$. Therefore, $h_{t}$ and $x^{b c}-y^{a}$ have the same knot of singularity by a result of Kouchnirenko [12, Corollaire 1.22].

Remark 24 Proposition 23 gives an algebraic proof of an observation by Baader, which states that the cobordism distance of $T(a, b c)$ and $T(b, a c)$ is equal to

$$
\frac{b c+a-a c-b}{2}=\frac{(b-a)(c-1)}{2}
$$

and which is a key proposition in [3].

Proposition 23 shows that if we define an algebraic counterpart of $c(a, b)$ in Section 5 , it is larger or equal to $\frac{b}{a}$, whereas in the Gordian setting $c(a, b)$ is smaller or equal to $\frac{b}{a}$ by Proposition 18. Thus, asymptotically, whenever $T(a, n) \leq_{g} T(b, m)$ for $a \leq b$ we get roughly $n \leq \frac{b}{a} m$ and, therefore, $T(a, n) \leq_{a} T(b, m)$. We take this as evidence to conjecture that for torus knots Gordian adjacency implies algebraic adjacency.

\section{References}

[1] V I Arnold, Normal forms of functions near degenerate critical points, the Weyl groups $A_{k}, D_{k}, E_{k}$ and Lagrangian singularities, Funkcional. Anal. i Priložen. 6 (1972) 3-25 MR0356124

[2] S Baader, Unknotting sequences for torus knots, Math. Proc. Cambridge Philos. Soc. 148 (2010) 111-116 MR2575377

[3] S Baader, Scissor equivalence for torus links, Bull. Lond. Math. Soc. 44 (2012) 10681078 MR2975163

[4] M Borodzik, C Livingston, Semigroups, $d$-invariants and deformations of cuspidal singular points of plane curves arXiv:1305.2868

[5] E Brieskorn, Beispiele zur Differentialtopologie von Singularitäten, Invent. Math. 2 (1966) 1-14 MR0206972

[6] E Brieskorn, H Knörrer, Plane algebraic curves, Modern Birkhäuser Classics, Birkhäuser, Basel (1986) MR2975988

[7] D B A Epstein, Curves on 2-manifolds and isotopies, Acta Math. 115 (1966) 83-107 MR0214087

[8] J-M Gambaudo, É Ghys, Braids and signatures, Bull. Soc. Math. France 133 (2005) 541-579 MR2233695 
[9] C M Gordon, R A Litherland, K Murasugi, Signatures of covering links, Canad. J. Math. 33 (1981) 381-394 MR617628

[10] F Hirzebruch, Singularities and exotic spheres, from: "Séminaire Bourbaki 1966-1968 (Exposé 314)", Soc. Math. France, Paris (1995) 13-32 MR1610436

[11] A Kawauchi, A survey of knot theory, Birkhäuser, Basel (1996) MR1417494

[12] A G Kouchnirenko, Polyèdres de Newton et nombres de Milnor, Invent. Math. 32 (1976) 1-31 MR0419433

[13] P B Kronheimer, T S Mrowka, Gauge theory for embedded surfaces, I, Topology 32 (1993) 773-826 MR1241873

[14] J Levine, Knot cobordism groups in codimension two, Comment. Math. Helv. 44 (1969) 229-244 MR0246314

[15] C Livingston, Computations of the Ozsváth-Szabó knot concordance invariant, Geom. Topol. 8 (2004) 735-742 MR2057779

[16] J Milnor, Singular points of complex hypersurfaces, Annals of Mathematics Studies 61, Princeton Univ. Press (1968) MR0239612

[17] H Murakami, Some metrics on classical knots, Math. Ann. 270 (1985) 35-45 MR769605

[18] K Murasugi, On a certain numerical invariant of link types, Trans. Amer. Math. Soc. 117 (1965) 387-422 MR0171275

[19] J Rasmussen, Khovanov homology and the slice genus, Invent. Math. 182 (2010) 419-447 MR2729272

[20] D Rolfsen, Knots and links, Mathematics Lecture Series 7, Publish or Perish, Houston, TX (1990) MR1277811

[21] D Siersma, Classification and deformation of singularities, University of Amsterdam, Amsterdam (1974) MR0350775

[22] L D Tráng, C P Ramanujam, The invariance of Milnor's number implies the invariance of the topological type, Amer. J. Math. 98 (1976) 67-78 MR0399088

[23] A G Tristram, Some cobordism invariants for links, Proc. Cambridge Philos. Soc. 66 (1969) 251-264 MR0248854

[24] H F Trotter, Homology of group systems with applications to knot theory, Ann. of Math. 76 (1962) 464-498 MR0143201

[25] H Wendt, Die gordische Auflösung von Knoten, Math. Z. 42 (1937) 680-696 MR1545700

Universität Bern

Sidlerstrasse 5, CH-3012, Bern, Switzerland

peter.feller@math.unibe.ch

Received: 21 March 2013 Revised: 21 August 2013 